\title{
Analysis of Steel Fiber Randomly Distributed Concrete Beams Based on ABAQUS
}

\author{
Zhu Kangwei, Xu Zhihong, Cao Zhiwei, Xu Wentao \\ Department of Civil Engineering, Nanjing University of Science and Technology, Nanjing, China
}

Email address:

2059201694@qq.com (Zhu Kangwei)

\section{To cite this article:}

Zhu Kangwei, Xu zhihong, Cao zhiwei, Xu wentao. Analysis of Steel Fiber Randomly Distributed Concrete Beams Based on ABAQUS. Science Discovery. Vol. 5, No. 7, 2017, pp. 592-597. doi: 10.11648/j.sd.20170507.30

Received: November 14, 2017; Accepted: November 23, 2017; Published: December 29, 2017

\begin{abstract}
In this paper, the stochastic distribution of steel fiber in concrete beam is realized by using MATLAB modeling software. Firstly, MATLAB software is used to establish random distribution of steel fibers of different number, length and position, and the steel fiber model is assembled in an embedded way with the concrete model. Then the ABAQUS software is used to carry out the three point bending monotonic loading test on the model, and the stress curves and deflection curves of different steel fiber concrete are obtained. The effect of steel fiber on tensile and crack resistance in concrete is analyzed. The effect of volume, length and location of steel fiber on stress, strain and deflection of concrete beam is studied. The results show that the increase of the volume fraction can decrease the deflection and increase the tensile strength of the material, while the length has no significant effect. The distribution can significantly affect the efficiency of the fiber.
\end{abstract}

Keywords: Steel Fiber Reinforced Concrete, Abaqus, Random Distribution

\section{基于ABAQUS的钢纤维随机分布混凝土梁分析}

朱康伟, 徐志洪, 曹志威, 许文涛

理学院土木工程系, 南京理工大学, 南京, 中国

邮箱

2059201694@qq.com（朱康伟）

摘要: 本文利用matlab建模软件实现了钢纤维在混凝土梁中的随机分布。首先通过matlab建立随机分布的不同数量、不 同长度、不同位置的钢纤维, 将得到的钢纤维模型以嵌入的方式与混凝土模型组装成整体。然后使用abaqus软件对模 型进行三点弯单调加载试验, 采集到不同钢纤维混凝土的应力曲线、挠度曲线。分析了钢纤维在混凝土中起到抗拉阻 裂作用,钢纤维体积率、长度、分布位置对混凝土梁的应力应变、挠度的影响。结论表明: 体积率增大可降低挠度, 提 高材料抗拉强度；长度的影响不显著；分布位置可以显著影响纤维的作用效率。

关键词：钢纤维混凝土, ABAQUS, 随机分布

\section{1. 引言}

水泥自问世以来, 各种改性混凝土的发明应用层出不

穷, 高强度混凝土是混凝土材料发展的一个重要方向。钢
纤维混凝土(Steel Fiber Reinforeed Conerete)是指在普通混 凝土中掺入纵向随机分布的钢纤维而形成的一种具有优 良力学性能的复合材料。随机分布的短钢纤维能对混凝土 性能起到很大改善作用, 它能阻碍混凝土内部微裂缝的发 展及宏观裂缝的发生和发展; 不仅能够提升混凝土的抗拉 
强度、抗弯强度、抗剪强度, 还提升了混凝土的延性、耐 久性能等。在有抗爆、抗冲击、抗裂等实际工程中有着良 好的应用前景。

近年来, 众多学者对钢纤维混凝土进行各种静力和动 力试验[2]-[3], 如钢纤维的静力损伤及疲劳试验、抗拉试 验[1] [4], 以及大量的有限元数值仿真 [5] [6]。西安建筑科 技大学邱继生 [7]在钢纤维混凝土的仿真试验中, 采用三种 方法考虑钢纤维的仿真模型。分别为修正混凝土本构关系 法、单元等效力筋法、整体等效力筋法, 仿真结果与实验 结果比较接近而成为一种可行的方法。然而上述三种方法 中, 前者改变本构关系简单实用却不能体现钢纤维的阻裂 效果, 后者得到的是近似的结果, 均不能准确反映随机钢 纤维散布在混凝土的增强效果。孙玉军 [8]采用先建立正六 面体单元, 然后随机抽出一部分作为钢纤维单元, 其他部 分作为混凝土单元。实现了随机性, 但并没有分离建模。 本文使用matlab建立随机分布钢纤维实体, 以嵌入方式与 混凝土共同工作，弥补了上述采取等效方法带来的问题， 研究钢纤维体积率、长度、分布位置三个因素对混凝土梁 抗拉性能、变形性能的影响。对钢纤维的改性性能研究有 着重要意义。

\section{2. 随机分布钢纤维混凝土梁有限元模型建立}

\section{1. 随机分布钢纤维模型建立}

钢纤维由于数量多、体积小, 在abaqus中直接建立实 体圆筒模型较为困难、不切实际。因此本文借助matlab批 量产生一定数量的细小长方形实体, 导入ansys 软件中, 生 成三维模型, 最后转入abaqus中扫描成型。

(1) 钢纤维随机生成

本文利用matlab强大的数学建模功能, 实现钢纤维分 布的随机性和不重叠型, 过程如图1所示。首先利用Rand 函数随机生成随机数组 (初始点) $\left(\mathrm{x}_{\mathrm{i}}, y_{i}, z_{i}\right)$, 将后生成 的数组 $\left(\mathrm{x}_{j}, y_{j}, z_{j}\right)$ 与前者相减, 如果得到的 $\mathrm{dx} 、 \mathrm{dy}$ 大 于宽和高或者 $\mathrm{dz}$ 大于长, 则后生成数组无效, 否则有效。 有效数组 (初始点数) 达到钢纤维数量时候终止, 将所有 的有效数组加上相应的长、宽、高得到长方体的另外 $7 \mathrm{n}$ 个点坐标。至此, 所有钢纤维三维坐标点建立完毕。

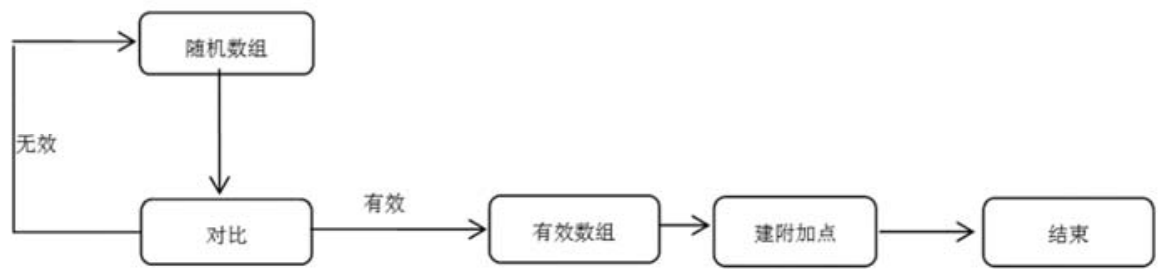

图1 Matlab建模流程图。

(2) 钢纤维实体成型

将matlab生成的三维坐标点放入文本文件中, 加入循 环语句, ansys软件读取后即进入循环, 实现由点连线、由 线构面、由面构体, 生成如图2所示的钢纤维的三维实体, 将生成文件以part实体的模式再扫描入abaqus中，生成最 终模型。
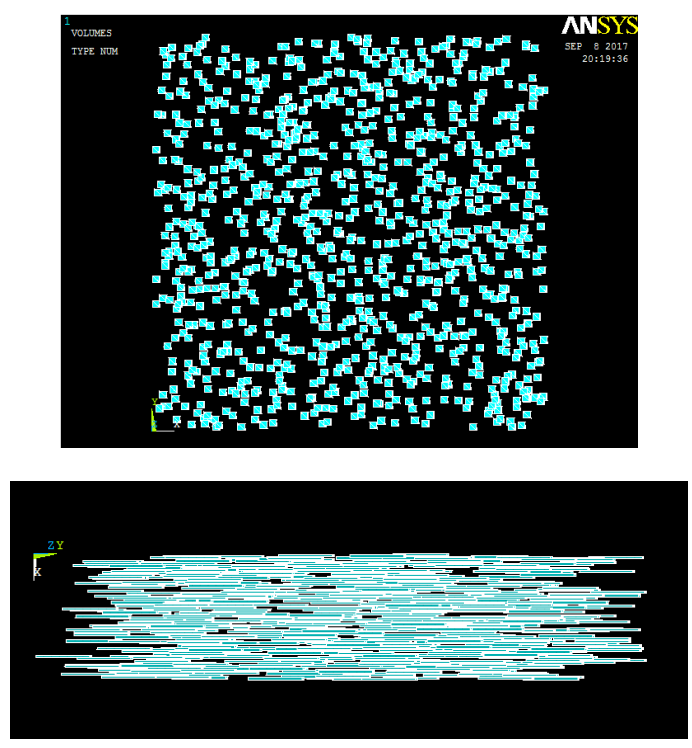

图2 ANSYS钢纤维3D模型。

\section{2. 有限元模型概况}

\subsection{1. 几何模型}

ABAQUS有限元模型分为混凝土和钢纤维两个部分。 在abaqus中直接建立混凝土模型如图所示, 混凝土梁尺寸 为 $40 \mathrm{mmx} 40 \mathrm{mmx} 300 \mathrm{~mm}$ 。梁两端简支梁支承, 跨中位置作 用集中力。钢纤维模型为了使其做到随机分布在混凝土试 件中, 使用Matlab软件进行编程（循环随机程序）, 采用 等效面积法把圆截面钢纤维换算成边长为 $0.6 \mathrm{~mm}$ 正方形 截面的钢纤维, 垫块采用 $20 \mathrm{mmx} 20 \mathrm{mmx} 40 \mathrm{~mm}$ 长方体钢材。 模型建立如图3所示。采用控制变量法控制钢纤维数量、 长度、位置的变化, 共建立 11 个钢纤维混凝土梁的模型, 结果如表1所示。

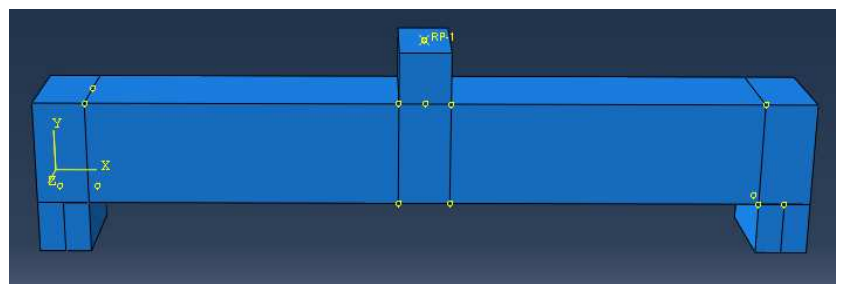

图3 ABAQUS模型。 
表1 钢纤维参数。

\begin{tabular}{|c|c|c|c|c|c|c|c|c|c|c|c|}
\hline & $\mathbf{A 8 0 0}$ & $\mathbf{A 1 0 0 0}$ & $\mathbf{A 1 5 0 0}$ & $\overline{\mathbf{A 2 0 0 0}}$ & B800 & $\mathbf{C 8 0 0}$ & D800 & 0 & A50-1 & A50-2 & A50-3 \\
\hline 长度 & 30 & 30 & 30 & 30 & 45 & 55 & 65 & 0 & 30 & 30 & 30 \\
\hline 体积率 & $1.8 \%$ & $2.25 \%$ & $3.38 \%$ & $4.5 \%$ & $2.7 \%$ & $3.3 \%$ & $3.9 \%$ & $0 \%$ & $1.8 \%$ & $1.8 \%$ & $1.8 \%$ \\
\hline 位置 & 随机 & 随机 & 随机 & 随机 & 随机 & 随机 & 随机 & 随机 & 受拉区 & 受压区 & 随机 \\
\hline
\end{tabular}

\subsection{2. 材料模型}

（1）混凝土模型

混凝土的密度为 2400 , 杨氏弹性模量为 $2.95 \mathrm{e} 10$, 泊松比 为 0.2 。 ABAQUS软件有三种混凝土本构模型[9], 包括混凝 土损伤塑性模型、混凝土弥散裂缝模型和和混凝土开裂模型。 本文使用的是混凝土损伤塑性模型。该模型考虑了混凝土在 拉压状态下的内部损伤, 既适用于静力分析也适用于动力分 析, 可以较好的描述混凝土的非弹性行为。混凝土损伤塑性 模型的塑性属性为剪切角取 $30^{\circ}$, 流动势偏移值取 0.1 , 双轴 极限抗压强度与单轴极限抗压强度的比值取 1.16 , 拉伸子午 面上和压缩子午面上的第二应力不变量比值取 0.6667 。为了 使损伤结合各向同性拉伸和压缩塑性, 在压缩行为

(Compressive Behavior) 和拉伸行为 (Tensile Behavior) 输 入如图4数据。完成了混凝土本构模型的建立。

\begin{tabular}{|c|c|c|c|c|}
\hline \multicolumn{2}{|c|}{ Plasticity } & \multicolumn{2}{|c|}{ Compressive Behavior } & Tensile Behavior \\
\hline \multicolumn{5}{|c|}{$\square$ Use strain-rate-dependent data } \\
\hline \multicolumn{5}{|c|}{$\square$ Use temperature-dependent data } \\
\hline \multicolumn{3}{|c|}{ Number of field variables: } & \multicolumn{2}{|c|}{$0 \stackrel{\Delta}{\frac{\Delta}{v}}$} \\
\hline \multicolumn{5}{|c|}{ Data } \\
\hline & & $\begin{array}{l}\text { ield } \\
\text { tress }\end{array}$ & $\begin{array}{l}\text { Ielastic } \\
\text { Strain }\end{array}$ & \\
\hline 1 & 182 & 00000 & 0 & \\
\hline 2 & 221 & .00000 & 0.0004 & \\
\hline 3 & 240 & 00000 & 0.0008 & \\
\hline 4 & 245 & 00000 & 0.0012 & \\
\hline 5 & 230 & 00000 & 0.002 & \\
\hline 6 & 216 & 00000 & 0.0024 & \\
\hline 7 & 166 & 00000 & 0.0036 & \\
\hline 8 & 113 & 00000 & 0.005 & \\
\hline
\end{tabular}

(a) 压缩行为

\begin{tabular}{|c|c|c|c|c|}
\hline \multicolumn{2}{|c|}{ Plasticity } & \multicolumn{2}{|c|}{ Compressive Behavior } & \multirow[t]{2}{*}{ Tensile Behavior } \\
\hline Type: & \multicolumn{2}{|c|}{ Strain } & & \\
\hline \multicolumn{5}{|c|}{$\square$ Use strain-rate-dependent data } \\
\hline \multicolumn{5}{|c|}{$\square$ Use temperature-dependent data } \\
\hline \multicolumn{3}{|c|}{ Number of field variables: } & \multicolumn{2}{|c|}{$0 \stackrel{\Delta}{\Delta}$} \\
\hline \multicolumn{5}{|l|}{ Data } \\
\hline & & $\begin{array}{l}\text { Yield } \\
\text { tress }\end{array}$ & $\begin{array}{l}\text { Cracking } \\
\text { Strain }\end{array}$ & \\
\hline 1 & & 00000 & 0 & \\
\hline 2 & & 60000 & 0.0001 & \\
\hline 3 & & 00000 & 0.0003 & \\
\hline 4 & & 90000 & 0.0004 & \\
\hline 5 & & 80000 & 0.0005 & \\
\hline 6 & & 23000 & 0.0008 & \\
\hline 7 & & 84000 & 0.001 & \\
\hline
\end{tabular}

(b) 拉伸行为

图4 混凝土压缩属性和压缩属性。
(2) 钢纤维模型

钢纤维质量密度为 7800 , 弹性模量为 $1.9 \mathrm{e} 11$, 泊松比 为 0.3 。钢纤维的本构模型与普通钢筋相似, 采用经典双 线性随动强化模型, 即使用双线性关系的应力应变曲线, 考虑了包辛格效应, 适用于本文的钢纤维模型。

\subsection{3. 模型的组装、参数设定、加载制度、网格划分}

\section{（1）模型的组装}

钢纤维和混凝土的组装有三种形式, 分别为钢纤维随 机分布、随机受拉区分布、随机受压区分布三种。在两种 材料的结合面采用ABAQUS提供的Embeded模式进行自 由度耦合, 将钢纤维设置为嵌入部分, 其余整体作为主区 域。垫块与混凝土间设置为绑定模式。两端垫块下设置为 铰接约束。

\section{(2) 加载制度}

本次仿真试验为钢筋混凝土梁的三点弯试验, 在梁的 跨中施加集中荷载。采用控制力加载法, 加载速度为 $0.05 \mathrm{KN} / \mathrm{s}$, 荷载最大值为 $14 \mathrm{KN}$ 。

(3) 网格划分

常见的有限元模型处理方式包括分离式、整体式和组 合式。本文混凝土和钢纤维采取分离式建模。混凝土采用 C3D8R八结点线性六面体缩减积分单元, 网络划分技术采 用结构化划分技术, 将混凝土梁划分为完全由六面体组成 的网格。

钢纤维由于结构复杂、数量众多、模型分割过程过于 复杂, 采用 $\mathrm{C} 3 \mathrm{D} 10$ 十结点二次四面体单元, 划分技术使用 自由网格划分技术，算法为Use mapped tri meshing on bounding faces where appropriate, 如果映射网格划分能够 提升边界面网格质量, 则在边界面使用映射网格划分代替 自由网格划分。钢纤维网格划分如图5所示。

图5 钢纤维网格划分。

\section{ABAQUS数据结果分析}

\section{1. 钢纤维体积率的影响}

本文设计了编号为0、A800、A1000、A1500、A2000 的五个不同钢纤维率的混凝土梁模型 (编号中的数字代表 钢纤维数量）。控制其他因素相同的情况下, 采集了跨中 底部混凝土拉应力和挠度, 示意图如图6所示。取 $\mathrm{A}$ 单元混 凝土拉应力及挠度, 分析如下: 


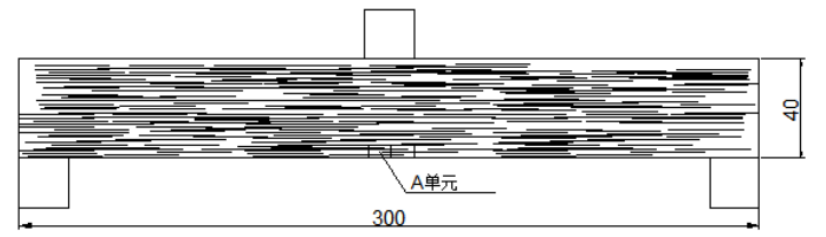

图6 A800模型示意图、

（1）从图7（a）、（b）可以明显看出, 混凝土拉 应力达到 $2.4 \mathrm{MP}$ 后进入塑性损伤状态, 与混凝土塑性损伤 模型相符合, 钢纤维的有无能够显著影响混凝土的力学 特性: 在有无对比试验中, 拉区钢纤维的最大拉应力能 达到 $300 \mathrm{MP}$ [10], 能够有效减少混凝土的拉应力, 阻止 或者延缓裂缝的发生, 对比模型 0 和A 800 , 可以发现混凝 土的最大拉应力降低约 $0.4 \mathrm{MP}$, 降低幅度为 $12.6 \%$; 跨中 最大挠度降低 $0.5 \mathrm{~mm}$, 降低幅度为 $11 \%$ 。

(2) 综合两图不难看出, 随着钢纤维体积率的增加, 混凝土受到的拉应力逐渐变小, 跨中最大挠度逐渐下降。 这表明, 钢纤维数量增多能够增强混凝土梁的抗拉强度、 提升延性、阻碍裂缝的发生, 有效地改善混凝土的力学性 能。

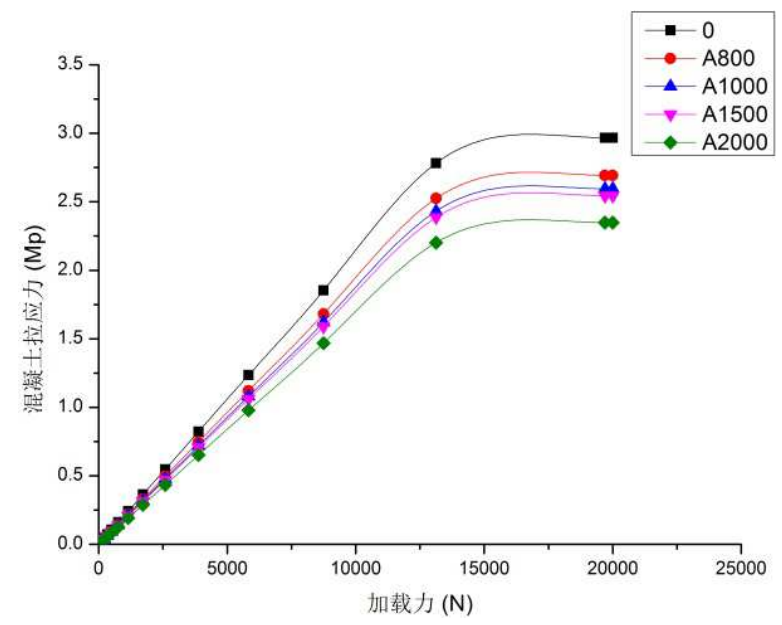

(a) 混凝土拉应力-加载力曲线

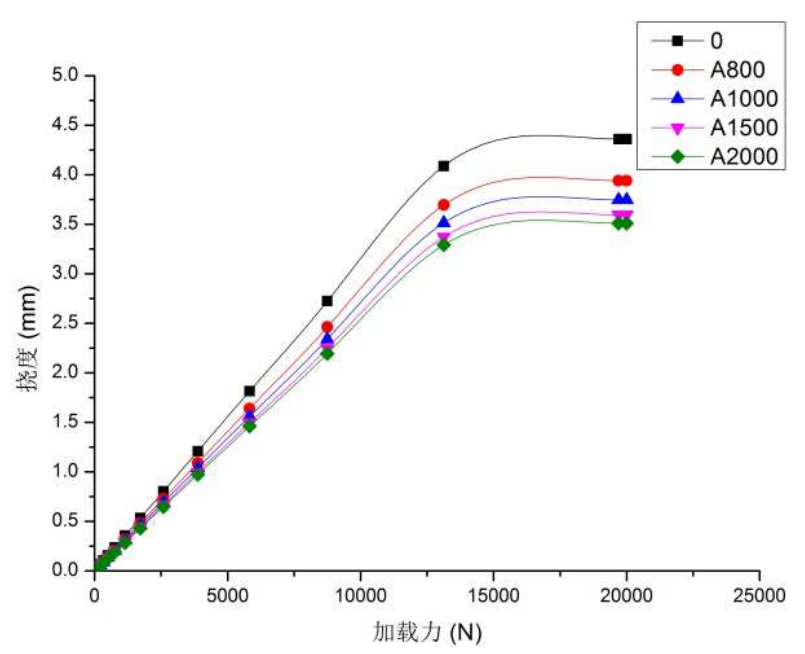

(b) 挠度-加载力曲线

图7 体积率的影响。

\section{2. 钢纤维分布位置的影响}

本文设计了编号为A50-1、A50-2、A50-3三个钢纤维 布置方式分别为受拉区、受压区、随机的混凝土梁模型, 如图8、9所示, 钢纤维数量均为 800 根。

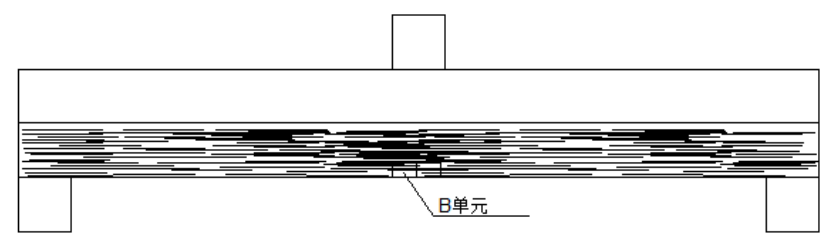

图8 受拉区布置示意图。

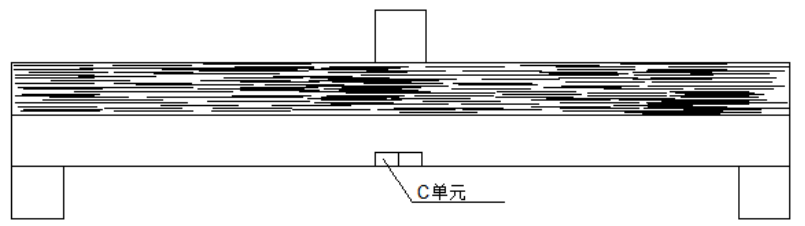

图9 受压区布置示意图。

取 $\mathrm{B} 、 \mathrm{C}$ 单元混凝土拉应力及挠度, 结合混凝土梁及 钢纤维模型的应力云图11、12。分析如下:

(1) 三种模型钢纤维均能降低混凝土单元拉应力, 不同的是布置在受拉区的钢纤维作用效果最好, 受拉区钢 纤维的应力也最大, 拉应力在 $300 \mathrm{MP}$ 以上; 而在受压区布 置及随机布置的模型, 混凝土拉应力基本相同, 受压区稍 差, 且钢纤维拉应力较低、压应力很大。三者均能有效降 低挠度，但作用效果三者基本相同。

（2）从应力云图中可以看出: 钢纤维在跨中受拉受 压区域相比其他区域较大, 与钢筋混凝土梁有类似的内力 性质, 而其他区域及跨中中和轴处钢纤维作用效果很低, 因此在应用钢纤维改善混凝土性质时候, 可以控制在跨中 拉压区布置、减少在其他位置的数量, 可以大大提升钢纤 维的利用效率。

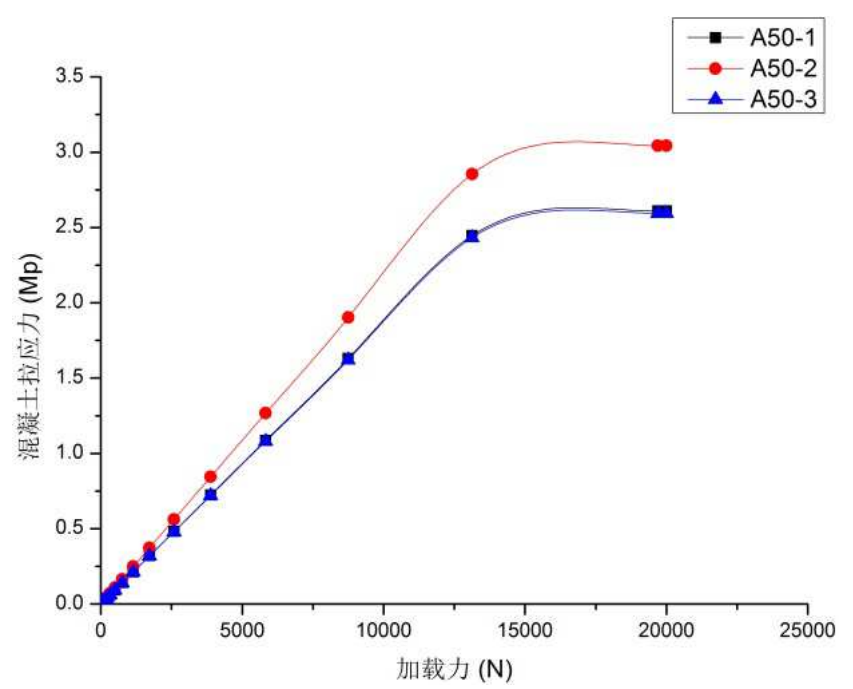

(a) 混凝土拉应力-加载力曲线 


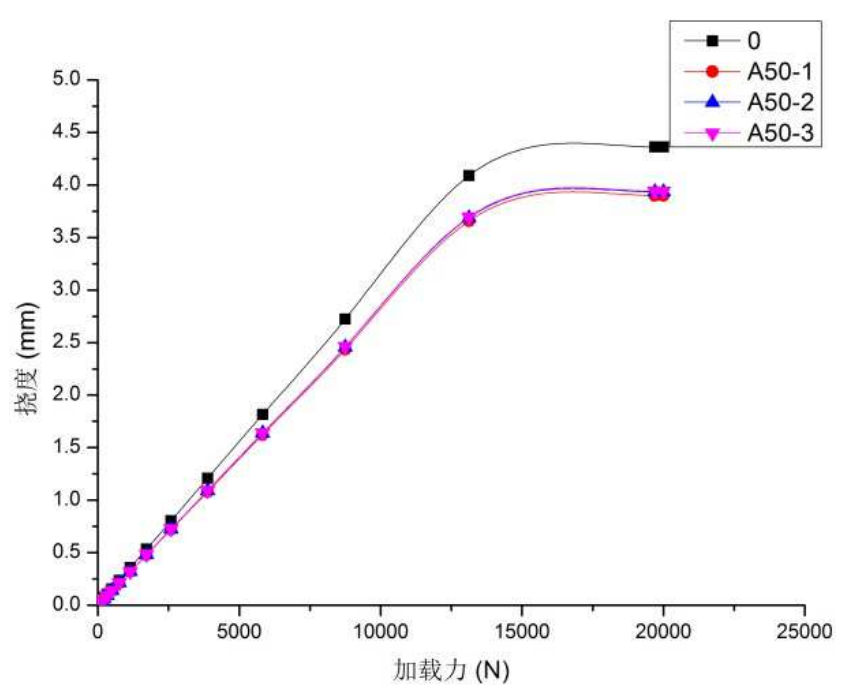

(b) 挠度-加载力曲线

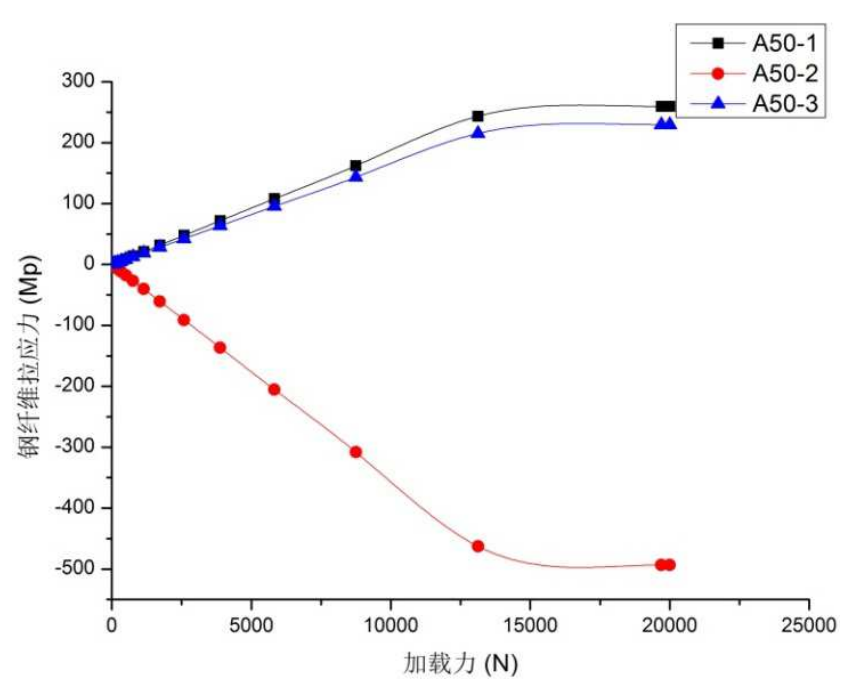

(c) 钢纤维拉应力-加载力曲线

图10 布置位置的影响。

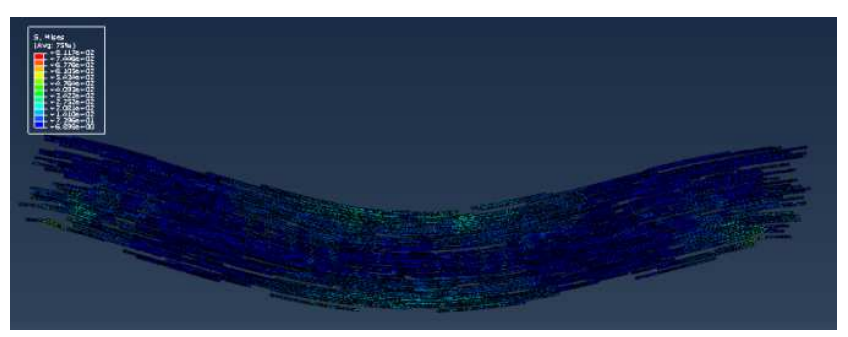

图11 钢纤维应力云图。

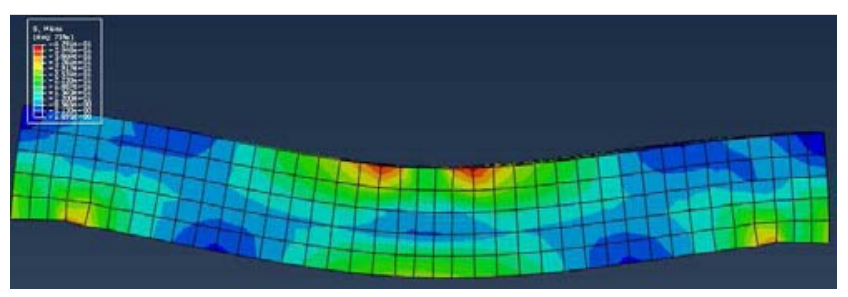

图12 混凝土应力云图。

\section{3. 钢纤维长度的影响}

本文设计了编号为 $0 、 \mathrm{~A} 800$ 、B $800 、 \mathrm{C} 800$ 、D 800 五 个钢纤维长度分别为 $30 \mathrm{~mm} 、 45 \mathrm{~mm} 、 55 \mathrm{~mm} 、 65 \mathrm{~mm}$ 的模型, 对比分析了不同长度 (长径比) 对作用效果的影响, 如图 13, 分析如下:

（1）四种不同长度的钢纤维均能有效降低混凝土拉 应力及跨中挠度, 效果明显。其中长度的变化对跨中挠度 的影响很小, 影响效果基本相同。但是对混凝土的拉应力 影响并不相同, 随着钢纤维数量的增加, 其对混凝土拉应 力的作用效果却降低, 降低幅度比较明显, 因此, 在选用 钢纤维长度, 应当考虑钢纤维最优长径比, 以免造成浪费、 降低效率。

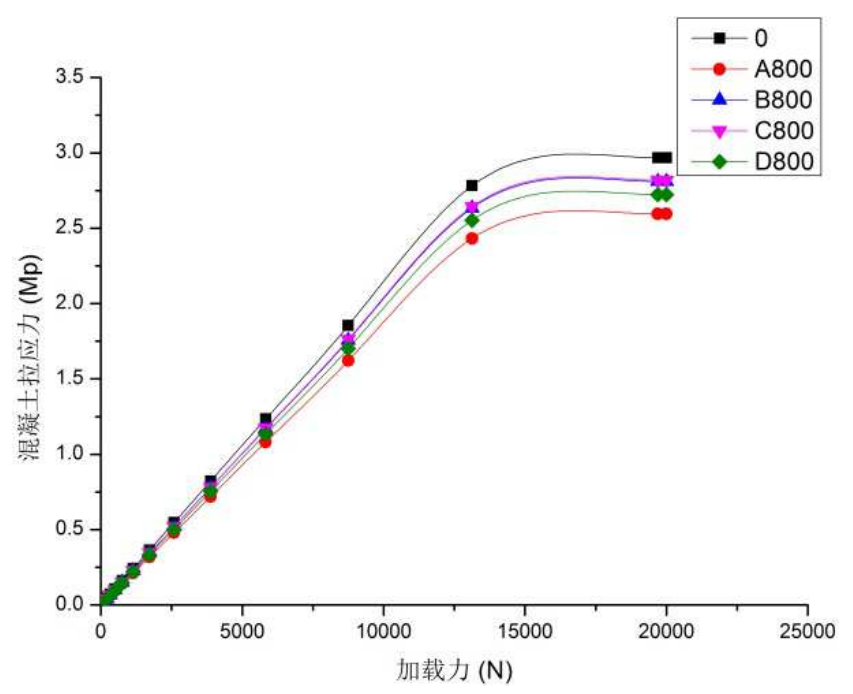

(a) 混凝土拉应力-加载力曲线

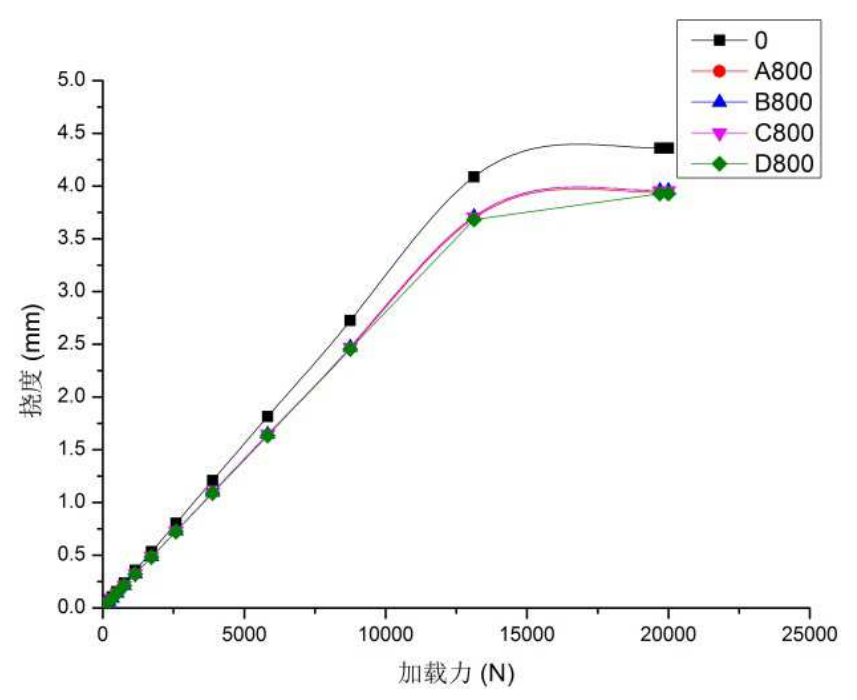

(b) 挠度-加载力曲线

图13 纤维长度的影响。

\section{4. 结论}

本文使用仿真软件能够有效模拟钢纤维随机分布及 钢纤维与混凝土在受力时候共同作用, 体现了钢纤维对混 
凝土的改性作用。钢纤维的体积率增加能有效提升混凝土 的抗拉强度, 阻碍裂缝的发生; 纤维长度对变形没有影响, 且大于 $30 \mathrm{~mm}$ 之后不利于混凝土的抗拉强度; 分布位置是 一个重要因素, 能够大大提升钢纤维的使用效率。

后续研究中, 应当进一步改进钢纤维与混凝土的粘结 模型为粘结一滑移模型，使仿真模型更接近现实情况。

\section{参考文献}

[1] 白敏, 牛荻涛, 姜否, 苗元耀. 钢纤维改善混凝土力学性能 和 微观结构的研究 [J]. 硅酸盐通报, 2013,32(10):2084-2089.[2017-09-10].

DOI:10.16552/j.cnki.issn1001-1625.2013.10.009。

[2] 杨润年. 钢纤维混凝土静力损伤及疲劳损伤研究 [D]. 华南 理工大学, 2013。

[3] Luigi Biolzi, Sara Cattaneo. Response of steel fiber reinforced high strength concrete beams: Experiments and code predictions $[\mathrm{J}]$. Cement and Concrete Composites, 2016, 34-42.

[4] G. A. Pikus. Steel Fiber Concrete Mixture Workability [J]. Procedia Engineering, 2016, 150 51-60.

[5] 关升. 钢纤维混凝土连续深梁有限元分析 [D]. 哈尔滨工程 大学, 200。
[6] 池寅, 徐礼华, 夏冬桃. 钢纤维混凝土深梁试验研究与非线 性有限元分析 [J]. 华中科技大学学报(城市科学版), 2007,(02):52-55+59。

[7] 邱继生. 钢纤维混凝土结构非线性有限元分析方法研究 [J]. 混凝土，2011,(03):17-20+24.[2017-09-14]。

[8] 孙玉军, Kwang Ik Kim, 张怀, 石耀霖. 利用有限元方法模 拟力学拉伸试验—以钢纤维混凝土为例 [J]. 地震, 2013,33(04):145-152. [2017-09-14]。

[9] 刘永胜, 王肖钧, 金挺, 劳俊. 钢纤维混凝土力学性能和本 构关系研究 [J]. 中国科学技术大学学报, 2007,(07):717-723。

[10] 韩嵘, 赵顺波, 曲福来. 钢纤维混凝土抗拉性能试验研究 [J]. 土木工程学报, 2006,(11):63-67。

\section{作者简介}

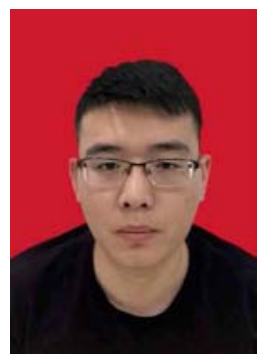

朱康伟, 江苏徐州, 理学院土木工程系, 南京理工大学, 所在城市: 南京。 